\title{
Expression of Leucine-rich Repeat-containing G-protein Coupled Receptor 5 and CD44: Potential Implications for Gastric Cancer Stem Cell Marker
}

ORIGINAL

ARTICLE

\section{Yoon Jin Choi ${ }^{1}$, Nayoung Kim ${ }^{1,2}$, Hye Seung Lee ${ }^{3}$, Seon Mee Park ${ }^{4}$, Ji Hyun Park ${ }^{2}$, Hyuk Yoon ${ }^{1}$, Cheol Min Shin ${ }^{1}$, Young Soo Park', Jin-Wook Kim ${ }^{1}$, Dong Ho Lee ${ }^{1}$}

${ }^{1}$ Department of Internal Medicine, Seoul National University Bundang Hospital, Seongnam, ${ }^{2}$ Department of Internal Medicine and Liver Research Institute, Seoul National University College of Medicine, Seoul, ${ }^{3}$ Department of Pathology, Seoul National University Bundang Hospital, Seongnam, ${ }^{4}$ Department of Internal Medicine, Chungbuk National University College of Medicine and Medical Research Institute, Cheongju, Korea

Background: The human leucine-rich repeat-containing G-protein coupled receptor (LGR) 5 and CD44 are one of the candidates for the marker of gastric cancer stem cells. We compared the expressions of two genes among control, dysplasia and cancer groups. Methods: We compared the mRNA expression of LGR5, CD44 and CD44v8-10 and immunohistochemistry (IHC) of LGR5 and CD44 in gastric antral mucosa of 45 controls, 36 patients with gastric dysplasia, and 39 patients with early gastric cancer. Additionally, IHC of LGR5 in gastric body mucosa was analyzed. Normal mucosa adjacent to dysplastic or cancer lesions was used for the quantitative real-time-PCR and IHC.

Results: Immunoreactivity of LGR5 in base of antral mucosa was higher in non-cancerous tissues of cancer than those of control $(P=$ 0.006), whereas the expression of LGR5 mRNA was not different among the three groups. Immunostaining of LGR5 was much stronger in the antrum than in the body of stomach $(P<0.001)$. Although there was no difference in antral immunointensity of LGR5 according to the severity of intestinal metaplasia, stronger immunostaining was found in the body with an aggravation of intestinal metaplasia $(P$ trend $<0.001)$. The expression of CD44v8-10 mRNA was higher in cancer patients than control subjects and patients with dysplasia $(P=0.018$ and 0.009$)$ while the expression of CD44 mRNA was higher in the control groups than the others.

Conclusions: IHC of LGR5 in crypt base and CD44 may be used for gastric CSC markers. LGR5 expression may be associated with the developing of corporal intestinal metaplasia. The expression of CD44v8-10 mRNA would be more suitable for gastric cancer stem cell marker than CD44 or LGR5 mRNA.

(J Cancer Prev 2016;21:279-287)

Key Words: LGR5 protein, Gastric cancer, Stem cell

\section{INTRODUCTION}

Gastric cancer remains the fourth most common cancer and the second leading cause of cancer-related mortality in the world. The treatment of gastric cancer remains a challenging problem because some patients with advanced gastric cancer who underwent a curative resection develop regional or distant recurrences. The overall 5-year survival rate for gastric cancer patients remains around $20 \%$ in the Western countries. ${ }^{1}$
The cancer stem cell (CSC) hypothesis that CSC may initiate carcinogenesis has emerged. Some data suggest that CSCs are present in many solid tumors and cancer cells are derived from a CSC compartment which undergoes an abnormal replicative process to form a non-stem cell component of the tumor. ${ }^{2}$ There has been growing evidence that Helicobacter pylori may promote emergence of CSCs in gastric cancer. ${ }^{3,4}$ The purification and characterization of CSCs could facilitate the identification of optimal targets for the better clinical outcomes, but discovery of

Received October 24, 2016, Revised November 14, 2016, Accepted November 15, 2016

Correspondence to: Nayoung Kim

Department of Internal Medicine, Seoul National University Bundang Hospital, 82 Gumi-ro 173 beon-gil, Bundang-gu, Seongnam 13620, Korea Tel: +82-31-787-7008, Fax: +82-31-787-4051, E-mail: nayoungkim49@empas.com, ORCID: Nayoung Kim, http://orcid.org/0000-0002-9397-0406

Copyright (C) 2016 Korean Society of Cancer Prevention

(c) This is an Open Access article distributed under the terms of the Creative Commons Attribution Non-Commercial License (http://creativecommons.org/licenses/by-nc/4.0) which permits unrestricted non-commercial use, distribution, and reproduction in any medium, provided the original work is properly cited. 
the marker for CSCs is the most important step.

Unfortunately, CSCs of gastric cancer have not yet been defined and characterized. Various CSC markers for gastric cancer such as CD44, ALDH1, LGR5, and CD166 were reported. ${ }^{5}$ Although CD44 has been used most frequently as a marker for CSCs in gastric cancer, previous studies have suggested that CD44v8-10, variant form of CD44 was a specific maker for gastric CSCs, but not CD44s. ${ }^{6.7}$ Leucine-rich repeat-containing G-protein coupled receptor 5 (LGR5) is an adult stem cell marker expressed in the small intestine, colon, stomach, and hair follicles. ${ }^{8}$ Currently, in vivo lineage tracing revealed that LGR5-postive cells at the base of the pyloric glands were multipotent stem cells that contributed to daily epithelial renewal. ${ }^{9}$

The technique of sorting cells based on cell surface marker expression and assessing their ability to initiate tumor growth in immunocompromised mice was a common method to investigate any possible existence of solid-organ CSCs. However, the clinical implication of measuring the expression of CD44 and LGR5 in human tissue has not been evaluated. Based on the above findings, we examined the potential application of evaluation of CD44 and LGR5 expression on human gastric mucosa in the normal-dysplasia-carcinoma sequence.

\section{MATERIALS AND METHODS}

\section{Study subjects}

Study subjects were consecutively enrolled at Seoul National University Bundang Hospital from February 2006 to December 2013. Subjects with a current $H$, pylori infection were participated. We divided subjects into 3 groups according to the pathologic findings, control, dysplasia, and early gastric cancer (cancer). Controls were defined as subjects with no evidence of malignant lesions or peptic ulcer by upper gastrointestinal (GI) endoscopy. Subjects with dysplasia or cancer group underwent endoscopic submucosal dissection or mucosal resection for treatment. Advanced gastric cancer was excluded since the aim was to assess early stages of carcinogenesis. All subjects completed a questionnaire with questions regarding demographic information and socioeconomic habits.

The study protocol was approved by the Ethics Committee at Seoul National University Bundang Hospital (IRB number B1301/186-111). Written informed consent was obtained from all subjects.

\section{H. pylori test and eradication}

During upper GI endoscopy, specimens for H. pylori infection status were obtained from both the antrum and body in the stomach. Initial H. pylori infection status was determined based on modified Giemsa staining, culture, and rapid urease test (CLO test; Delta West, Bentley, Australia). ${ }^{10}$ If one of these tests was positive, the subjects were regarded to be currently infected by $H$. pylori. The degree of intestinal metaplasia (IM) was recorded using the updated Sydney scoring system $(0=$ none, $1=$ slight, $2=$ moderate, and $3=$ marked $).{ }^{11}$

The first-line therapy for $H$. pylori infection included 7-day triple therapy (esomeprazole $40 \mathrm{mg}$ bis in die [b.i.d.], clarithromycin $500 \mathrm{mg}$ b.i.d., and amoxicillin $1000 \mathrm{mg}$ b.i.d.). To evaluate whether $H$. pylori was eradicated, a ${ }^{13} \mathrm{C}$-urea breath test was performed at least 4 weeks after the completion of therapy. If $H$. pylori were not eradicated, 2 weeks of second-line treatment (tripotassium dicitrate bismuthate $300 \mathrm{mg}$ quater in die [q.i.d.], metronidazole $500 \mathrm{mg}$ ter in die, tetracycline $500 \mathrm{mg}$ q.i.d., and esomeprazole $20 \mathrm{mg}$ b.i.d.) was administered.

\section{Real-time quantitative PCR}

Total RNA was extracted directly from non-cancerous corporal biopsy specimens with TRIzol ${ }^{\mathbb{R}}$ reagent (Invitrogen, Carlsbad, CA, USA), as recommended by the manufacturer. Next, 1,000 ng of RNA was reverse transcribed to cDNA with oligo (dT) and Moloney murine leukemia virus reverse transcriptase (Invitrogen), according to the manufacturer's instructions. Quantitative PCR was performed in 96-well reaction plates using $2 \mu \mathrm{L}$ of $\mathrm{CDNA}$ in a $20 \mu \mathrm{L}$ reaction mix containing $2 \times$ SYBR $^{\circledR}$ Premix Ex Taq ${ }^{\mathrm{TM}}$ (Takara Bio Inc., Otsu, Japan). Samples were run on StepOnePlus real-time PCR instrument (Applied Biosystems, Foster City, CA, USA), The PCR cycling conditions were as follows: an initial denaturation step for 30 seconds at $95^{\circ} \mathrm{C}$ and then 40 cycles of denaturation at $95^{\circ} \mathrm{C}$ (5 seconds) and annealing at $60^{\circ} \mathrm{C}(34$

Table 1. Primer sequences for quantitative real-time-PCR

\begin{tabular}{llc}
\hline \multicolumn{1}{c}{ Gene name } & \multicolumn{1}{c}{ Primer } & Size (bp) \\
\hline h_LGR5_foward & CTC CCA GGT CTG GTG TGT TG & 149 \\
h_LGR5_reverse & GAG GTC TAG GTA GGA GGT & \\
& GAA G & \\
h_CD44s_forward & TGC CGC TTT GCA GGT GTA T & 66 \\
h_CD44s_reverse & GGC CTC CGT CCG AGA GA & \\
h_CD44v8-10_ & AGA ATC CCT GCT ACC AAT ATG & 334 \\
forward & GAC TC \\
h_CD44v8-10_ & AGG TCA CTG GGA TGA AGG TC \\
reverse & & \\
h_GAPDH_forward & TGT GGG CAT CAA TGG ATT & 115 \\
h_GAPDH_reverse & ACA CCA TGT ATT CCG GGT CAA T & \\
\hline
\end{tabular}


seconds with the final dissociation stage of 15 seconds at $95^{\circ} \mathrm{C}, 1$ minute at $60^{\circ} \mathrm{C}$, and 15 seconds at $95^{\circ} \mathrm{C}$. The primer sequences for PCR are shown in Table 1. Expression levels of mRNA of the target gene were compared with the endogenous control $\beta$-actin using the $2^{-\Delta \Delta \mathrm{Ct}}$ method. ${ }^{6}$

\section{Immunohistochemistry}

Immunohistochemistry (IHC) of CD44 and LGR5 was also performed. Basically, paraffin-embedded mucosal tissue distant from tumor sites was analyzed. For the evaluation of dysplasia and cancer, the normal mucosa of stomach part where the dysplasia or cancer was located (i.e., antrum or body) was used. Core tissue biopsies ( $2 \mathrm{~mm}$ in diameter) were obtained from paraffin-embedded gastric mucosa. Cores were arranged in recipient paraffin blocks (tissue array blocks) using a trephine. The test procedure used a human control slide for IHC analysis (Superbiochips Laboratories, Seoul, Korea). Antibodies against CD44 (H-CAM) (1:200 dilution; Leica Biosystems, Breckland, UK) and LGR5 (ab75732) (1:30 dilution; Abcam, Cambridge, UK) were used. Staining of sections ( $4 \mu \mathrm{m}$ thick) from tissue array blocks was performed using the BenchMark XT staining system and the ultraVIEW universal DAB detection kit (Ventana Medical Systems Inc., Tucson, AZ, USA). Aberrant expression of CD44 is observed in the membrane and cytoplasm of cancer cells. ${ }^{12}$ LGR5 was strongly expressed in the membrane of non-neoplastic glands. Immunointensity of LGR5 was evaluated separately at surface mucosa and base of crypt. Scoring for the expression of CD44 and LGR5 was determined using light microscopy, by multiplying the intensity times the area (\%) where staining was observed in epithelial glands; the possible scores ranged from 0 to $300 .{ }^{3}$ Area was defined as a 'stained cells in a gland/total cells in a gland'. More than 5 glands were evaluated. The intensity of staining was scored as 0 , no staining; $1+$ faint/barely perceptible partial staining; $2+$, weak to moderate staining; $3+$, strong staining. ${ }^{13}$ Tests were repeated three times and mean values were described. Each sample was scored by a blind reviewer.

\section{Statistical analysis}

The $\chi^{2}$ test and Fisher's exact test were used for the analysis of categorical variables The mRNA levels encoding CD44v8-10 and LGR5 markers were compared between groups using one-way ANOVA followed by Scheffe's or Tamhane's tests. For the analysis of changes in histologic grades or mRNA expression after the eradication of $H$. pylori, a paired $t$-test or Wilcoxon rank sum test was used. All analyses were performed using IBM SPSS ver. 21.0 (IBM, Armonk, NY, USA). $P$-values less than 0.05 were considered statistically significant.

\section{RESULTS}

\section{Demographic characteristics}

A total of 237 patients in whom H. pylori had been eradicated and who had been followed for more than 6 months were enrolled. The mean follow-up duration was 46.7 months (range, 6.0-112.4 months). Among them, 45 controls, 36 subjects with gastric dysplasia, and 39 subjects with early gastric cancer were randomly selected and analyzed due to limited fund. Antral mucosal tissue distant from tumor sites was analyzed from 30, 21, and 25 subjects in the control, dysplasia, and cancer groups, respectively for the evaluation of mRNA and IHC. For the additional evaluation of LGR5 IHC in body, body mucosae were used from 15 control and 15 dysplasia, and 14 cancer subjects with a lesion in gastric body. Demographic characteristics were listed in Table 2.

\section{Association between human leucine-rich repeat- containing G-protein coupled receptor 5 expression and gastric carcinogenesis}

The IHC scores of LGR5 were compared among control, dysplasia and cancer. While the IHC scores of surface of gastric mucosa did not show any differences (Fig. 1A), IHC scores of base of gastric mucosa from non-cancer tissues in cancer subjects were markedly upregulated than those in controls $(27.20 \pm 4.50$ vs.

Table 2. Clinicohistological characteristics of subjects whose antral mucosa were evaluated

\begin{tabular}{|c|c|c|c|c|}
\hline Clinicohistological characteristic & Controls $(\mathrm{n}=30)$ & Dysplasia $(\mathrm{n}=21)$ & Early gastric cancer $(\mathrm{n}=25)$ & $P$-value \\
\hline Men & $12(40.0)$ & $11(52.3)$ & $16(64.0)$ & 0.357 \\
\hline Age (yr) & $55.6 \pm 9.9$ & $60.9 \pm 7.5$ & $60.2 \pm 10.6$ & 0.104 \\
\hline Pepsinogen $\mathrm{I} / \mathrm{II}$ ratio & $2.70 \pm 0.26$ & $2.81 \pm 0.30$ & $2.59 \pm 0.17$ & 0.845 \\
\hline Atrophic gastritis & $0.82 \pm 0.16$ & $1.50 \pm 0.20$ & $1.05 \pm 0.22$ & 0.077 \\
\hline Intestinal metaplasia & $0.81 \pm 0.15$ & $1.35 \pm 0.24$ & $1.68 \pm 0.17$ & $0.002 *$ \\
\hline
\end{tabular}

Values are presented as number $(\%)$ or mean $\pm \mathrm{SE}$. *This value indicates clinical significance $(P<0.05)$. 
A

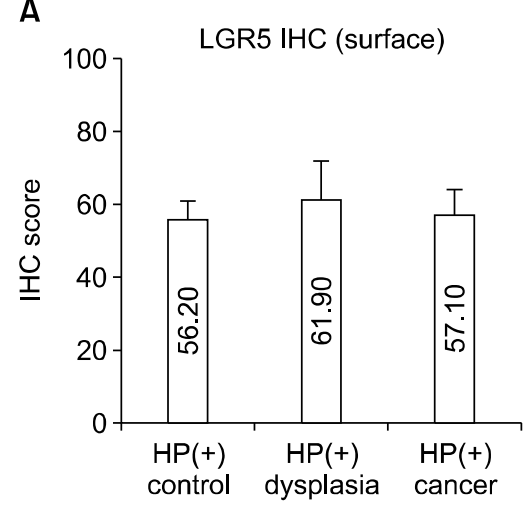

D

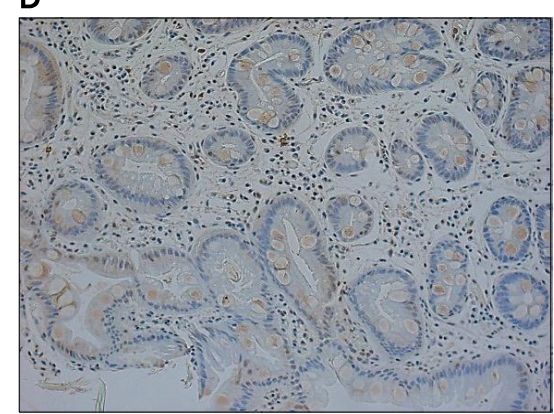

B

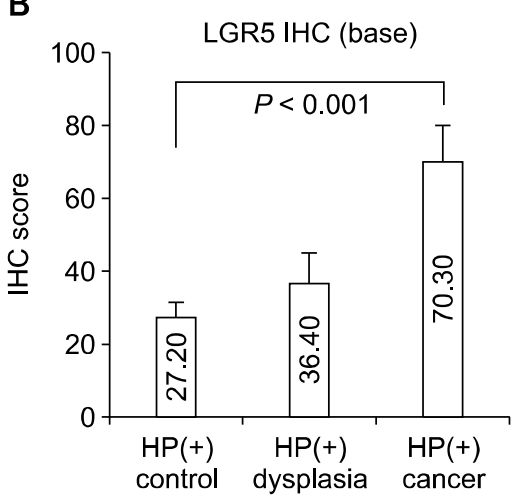

E

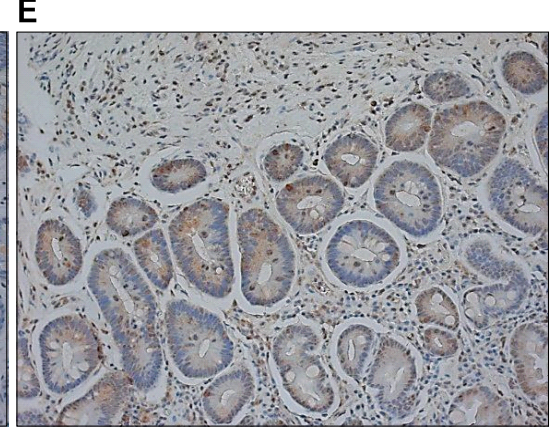

C

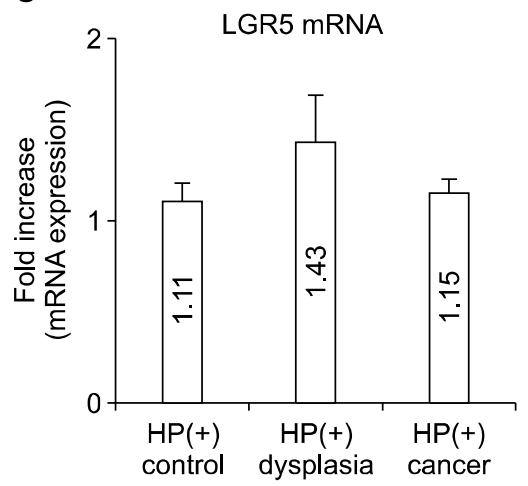

$\mathbf{F}$

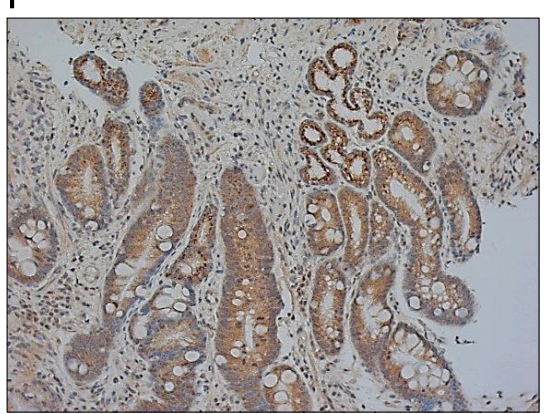

Figure 1. LGR5 expression according to normal-dysplasia-cancer sequence. (A, C) There is statistically significant difference in expression of neither surface LGR5 IHC or LGR5 mRNA, (B) while immunoreactivity of LGR5 (base) was higher in cancer than in control samples. (D-F) LGR5 immunostaining in base of gastric mucosa in representative control $(\times 200)$, (E) dysplasia, and $(F)$ cancer samples. Data shown are means \pm SE. HP, Helicobacter pylori; IHC, immunohistochemistry; LGR5, leucine-rich repeat-containing G-protein coupled receptor 5.

$70.30 \pm 9.70, P<0.001$ ) (Fig. 1B). However, there were no significant differences in LGR5 mRNA expression among the three groups (Fig. 1C). The IHC intensity of LGR5 was increased from control to cancer (control, $27.20 \pm 4.50$; dysplasia, $36.40 \pm 8.60$; and cancer, $70.30 \pm 9.70 ; P$ for trends $<0.001$ ) (Fig. 1D-1F).

\section{Effects of $H$. pylori eradication on the expression of human leucine-rich repeat-containing G-protein coupled receptor 5 expression}

We then evaluated the effects of $H$. pylori eradication on the IHC and mRNA expressions of LGR5. IHC was evaluated in the base of gastric mucosa. The IHC intensity of LGR5 was markedly reduced at approximately 1 year after $H$. pylori eradication ( $45.36 \pm 3.54$ vs. $32.45 \pm 2.82, P<0.001)$. To determine whether the change in LGR5 expression occurs regardless of the development of cancer or dysplasia, we examined the changes in LGR5 IHC after H. pylori eradication in the 3 groups. After H. pylori eradication, control and cancer groups showed a significant reduction in the expression of LGR5 IHC (control: $29.59 \pm 4.41$ vs. $15.25 \pm 2.43, P=0.004$; cancer: $72.73 \pm 7.01$ vs. $49.95 \pm 5.64, P=$ 0.004) (Fig. 2A and 2C). However, non-cancerous mucosa of $H$. pylori-positive subjects with dysplasia did not show significant changes of LGR5 IHC (Fig. 2B). In terms with the LGR5 mRNA expression, only marginal reduction in LGR5 mRNA expression in cancer group (Fig. 2F), while others did not show significant reduction (Fig. 2D and 2E).

\section{Association between CD44 expression and gastric carcinogenesis}

As a conventional marker for the gastric CSC, IHC staining of $\mathrm{CD} 44$ was analyzed. The cancer group showed higher IHC score of CD44 compared with the control group $(99.41 \pm 16.10$ vs. $14.30 \pm$ 4.18, $P<0.001$ ) (Fig. 3A).

When CD44s mRNA was evaluated, cancer or dysplasia groups showed lower expression of CD44s mRNA than control (0.48 \pm 0.14 and $0.24 \pm 0.04$ vs. $1.55 \pm 0.19$, all $P<0.001$ ) (Fig. 3B). The expression of CD44v8-10 mRNA was higher in cancer group than control or dysplasia group ( $P=0.009$ and $P<0.001$ ) (Fig. 3C).

The CD44 IHC and mRNA expressions of CD44s and CD44v8-10 were compared before and after H. pylorieradication. For CD44 IHC, the cancer group showed a marked reduction in expression after eradication and resection, while the differences 

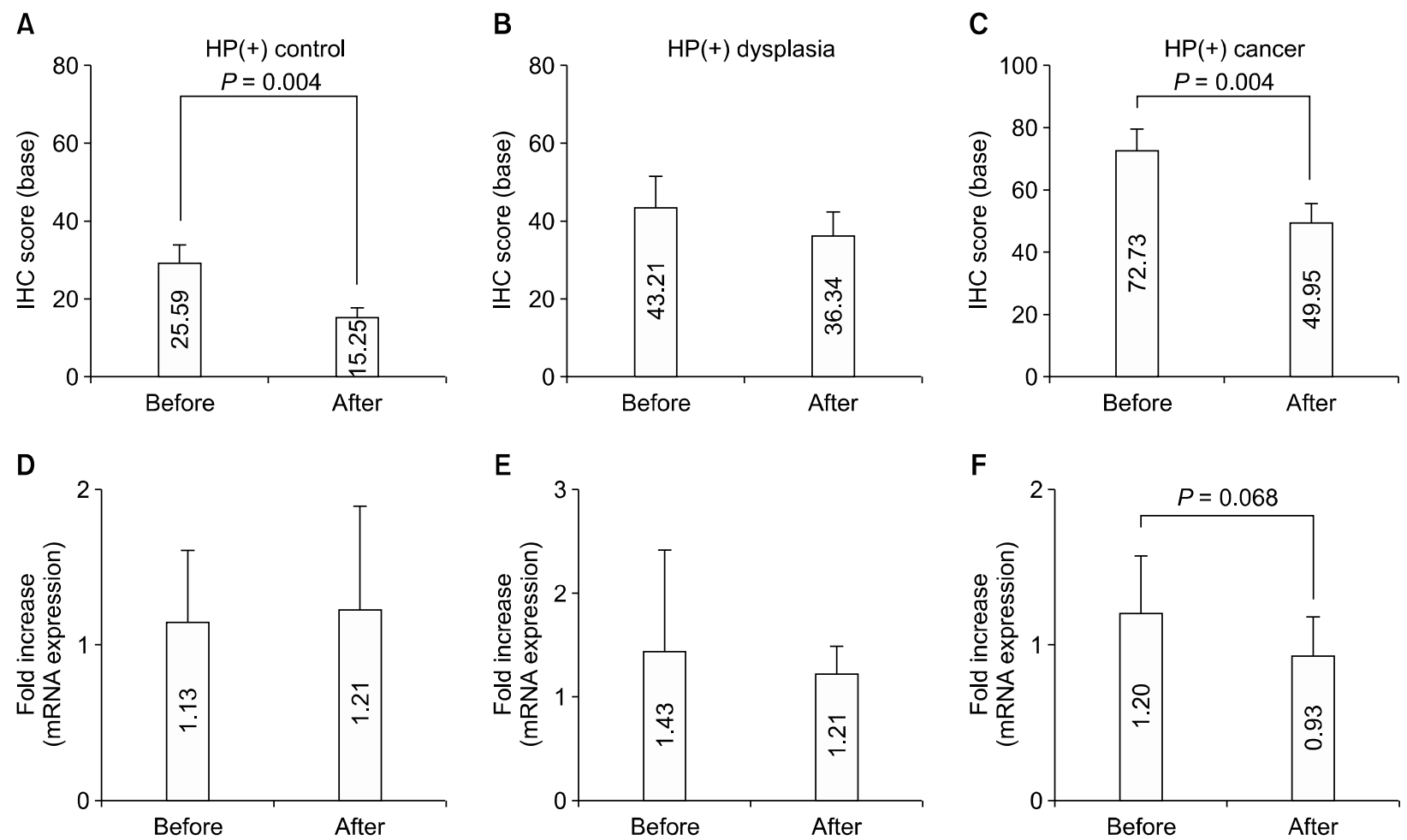

Figure 2. Effects of HP eradication on the LGR5 expression. After eradication, (A) control and (C) cancer groups showed a significant reduction in the expression of LGR5 IHC (base part). (B) Non-cancerous mucosa of HP-positive subjects with dysplasia showed no significant changes of LGR5 IHC. Regarding LGR5 mRNA expression, while there was no significant change in its mRNA expressions in subjects with (D) normal and (E) dysplasia, (F) there was only marginal reduction in LGR5 mRNA expression in cancer patients. Data shown are means \pm SE. HP, Helicobacter pylori; IHC, immunohistochemistry; LGR5, leucine-rich repeat-containing G-protein coupled receptor 5.

in the dysplasia and control groups did not reach statistical significance (control: $22.10 \pm 8.70$ to $20.31 \pm 8.10, P=0.304$; dysplasia: $60.70 \pm 10.51$ to $49.79 \pm 9.23, P=0.081$; cancer: $98.39 \pm 12.37$ to $45.68 \pm 7.72, P=0.013$ ) (Fig. 3D). There was no significant change of mRNA expression of CD44s or CD44v8-10 (Data not shown).

\section{Expression of human leucine-rich repeat-containing G-protein coupled receptor 5 immunohistochemistry according to the location of gastric mucosa and intestinal metaplasia}

IHC score of LGR5 in the body was significantly lower than in the antrum ( $29.45 \pm 4.40$ vs. $56.21 \pm 4.21, P<0.001)$ (Fig. $4 \mathrm{~A})$. While anturm did not show any significant difference in LGR5 IHC score according to severity of IM ( $P=0.637)$ (Fig. 4B), body mucosa with IM showed higher IHC score than that without IM (53.98 \pm 7.29 vs. 7.11 $\pm 2.17, P<0.001)$. LGR5 IHC score of gastric body showed an increasing trend according to the severity of IM (mild IM: $43.13 \pm 8.33$; moderate/severe IM: $57.60 \pm 9.29, P$ for trends $<0.001$ ) (Fig. 4C-4E).

\section{Association between expression of human leucine-} rich repeat-containing G-protein coupled receptor 5 and CD44

To investigate the relationship between LGR5 and CD44, the IHC scores were compared using Spearman's rho test. The IHC score of LGR5 significantly correlated with the IHC score of CD44 (surface of mucosa: Spearman's rho $=0.354, P<0.001$ ). However, there was no significant correlation between CD44 IHC and mRNA expression of CD44s and CD44v8-10. Moreover, LGR5 IHC scores did not correlate with the expression of LGR5 mRNA.

\section{DISCUSSION}

Immunoreactivity of CD44 and LGR5 at mucosal base progressively increased from normal to dysplasia to cancer. Following $H$. pylori eradication, there was a reduction in immunointensity of mucosal base LGR5 and CD44 in cancer patients. In the case of the cancer and control group with $H$. pylori-eradication after endoscopic resection of cancer, a marginal reduction in LGR5 IHC mRNA was detected. While expression of CD44s mRNA was 
A

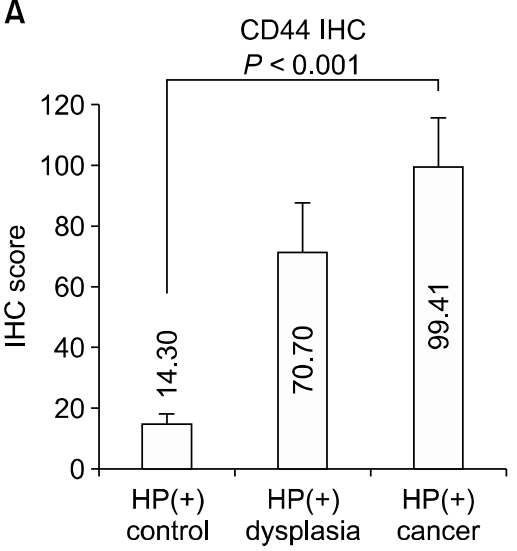

D

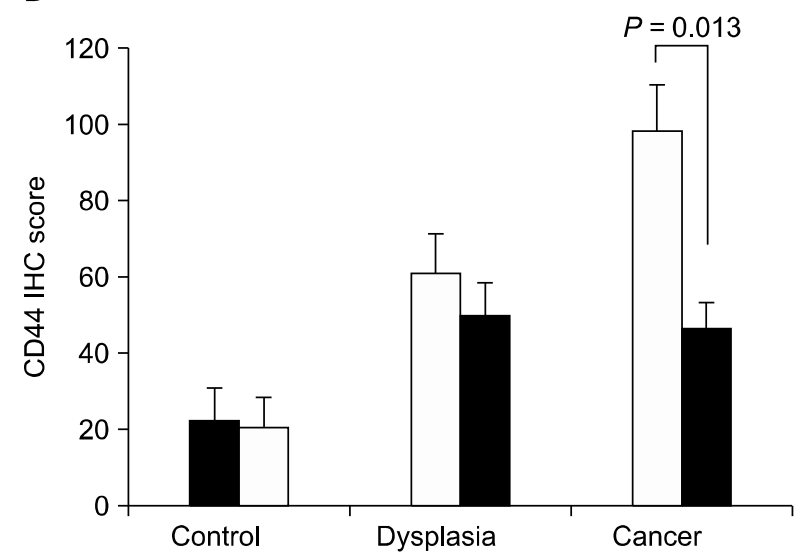

CD44 mRNA

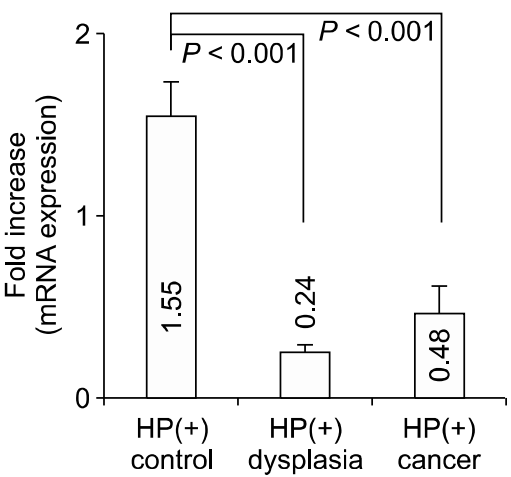

C

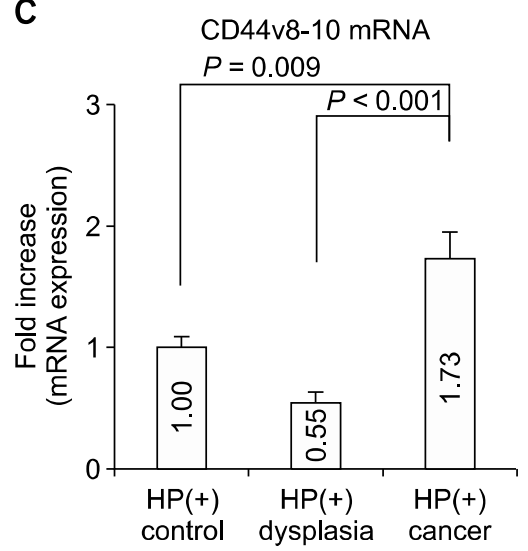

Figure 3. CD44 expression according to normal-dysplasia-cancer sequence. (A) Immunoreactivity of CD44 was higher in cancer than in control samples (A). The expression of CD44s mRNA was higher in the control groups than the others (B) while that of CD44v8-10 mRNA was higher in cancer patients than control subjects and patients with dysplasia $(P=0.009$ and $<0.001)$ (C). (D) Immunoreactivity scores of CD44 were analyzed with a paired $t$-test. The white bars indicate the initial score of immunostaining, whereas the black bars indicate staining at approximately 1 year after successful HP eradication in HP-positive subjects. Data shown are means \pm SE. HP, Helicobacter pylori; IHC, immunohistochemistry.

higher in control subjects than patients with dysplasia or cancer, that of CD44v8-10 mRNA was the highest in the cancer patients. Although IHC staining of CD44 correlated well with the expression of LGR5 immunoreactivity of mucosal base, there was no correlation between mRNA and IHC expression regarding either CD44 or LGR5. IHC score of LGR5 was progressively increased when the IM of body became severer.

American Association of Cancer Research Workshop made a definition of CSC as "cells within a tumor that possess the capacity for self-renewal and that can cause the heterogeneous lineages of cancer cells that constitute the tumor." ${ }^{14}$ Even though accumulative evidence supports the existence of CSCs that have the capacity to generate tumor, the stem cell hypothesis in carcinogenesis is under debate. A major obstacle for stem cell filed in solid tumor is that there is no specific tumor marker for CSC especially for stomach cancer.
Currently, in vivo lineage tracing revealed that a group of Lgr5-postive cells at the base of the pyloric glands were multipotent stem cells that contributed to daily epithelial renewal. ${ }^{9}$ LGR5 was proposed as an early diagnostic marker for gastric CSC, since upregulated expression of LGR5 was correlated with the gastric sequential cascades, "Correa pathway". ${ }^{15}$ Based on this observations we hypothesized that LGR5 may have an impact on carcinogenesis in the human stomach. However, investigations of LGR5 expression in human tissues have been limited, and an appropriate histological method to identify LGR5-positive cells in human organs has not been established. ${ }^{16}$ We tested whether the sequential changes of gastric carcinogenesis, i.e., normal, IM and invasive carcinoma, are associated with an expression of the LGR5 + cells. Although Simon et al. ${ }^{17}$ reported the LGR5 cells had different spatial distribution according to the 'precancerous cascade', we could not discriminate the immunointensity of 

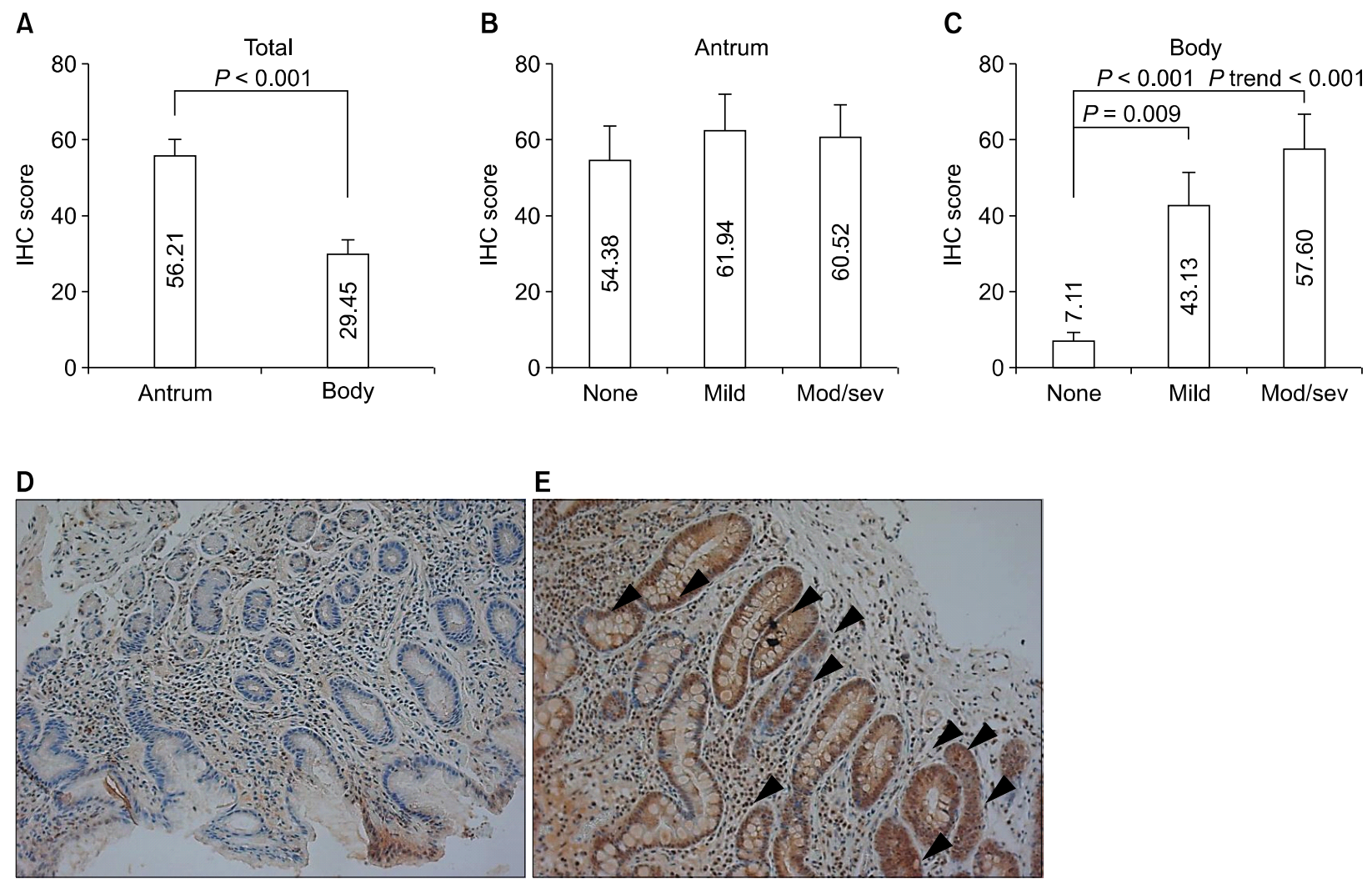

Figure 4. Immunoreactivity of LGR5 according to the location of gastric mucosa and IM. (A) LGR5 IHC in the body was significantly reduced than in the antrum. (B) Antral mucosa did not show any significant difference in LGR5 IHC according to presence or absence of IM. (C) Gastric body mucosa with IM showed an elevated immunointensity than in that without IM. The representative pictures of LGR5 stating of basal part of gastric body mucosa without IM $(\times 200)$ (D) and with severe IM $(\times 200)(\mathrm{E})$. Arrowheads membranous staining of LGR5. Data shown are means \pm SE. LGR5, leucine-rich repeat-containing G-protein coupled receptor 5; IM, intestinal metaplasia; IHC, immunohistochemistry; Mod/sev, moderate/severe.
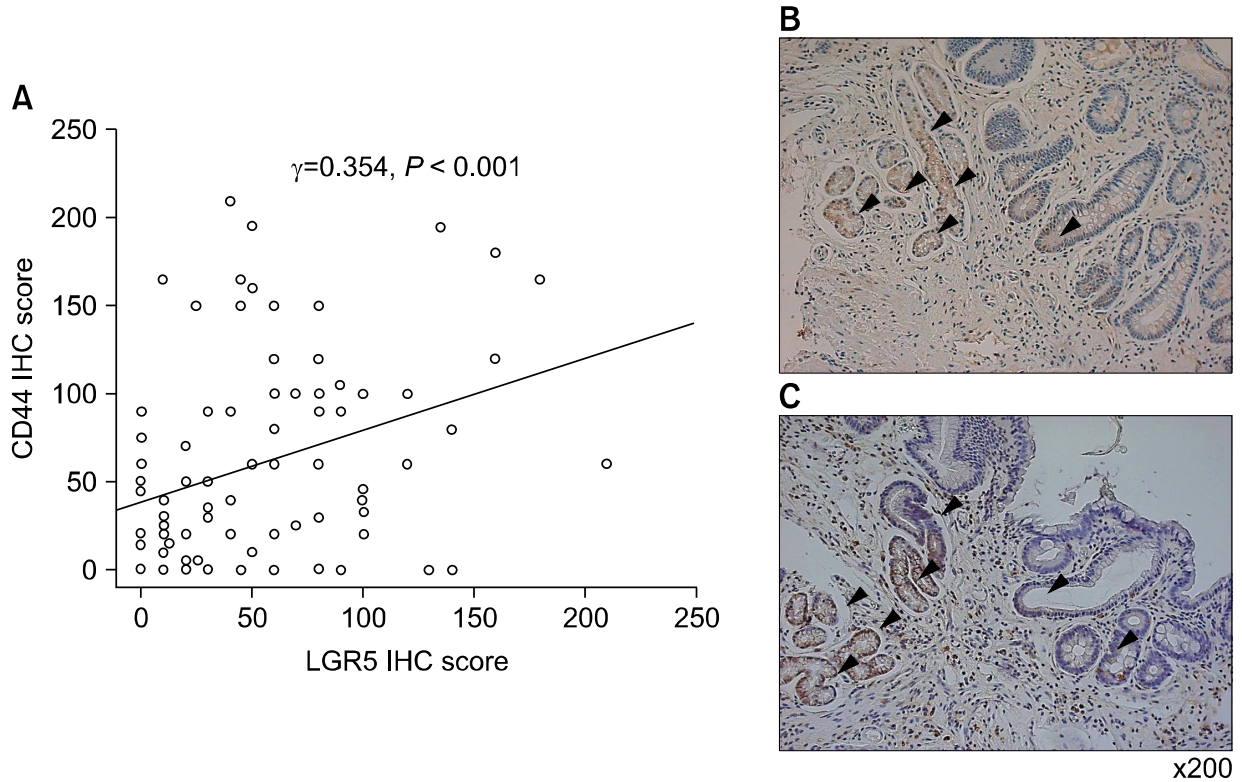

Figure 5. Association between the expression of LGR5 and CD44 immunohistochemical results. (A) There is a significant positive correlation between of LGR5 and CD44 immunohistochemical results. The distribution of cells stained with (B) CD44 ( $\times 200)$ and (C) LGR5 is similar in serial sections $(X 200)$. Arrowheads indicate membranous staining of CD44 and LGR5. IHC, immunohistochemistry; LGR5, leucine-rich repeat-containing G-protein coupled receptor 5. 
LGR5 in surface mucosa due to too diffuse and strong periplasmic expression. Therefore, since the usual storage of stem cells of gastric mucosa is crypt base, we measured the LGR5 immunointensity in the base of mucosa. In the present study, non-cancerous tissue of $H$. pylori-positive control, patients with dysplasia and cancer showed an increasing tendency of LGR5 immunoreativity in crypt base in accordance with CD44 IHC. Although double staining of LGR5 and CD44 was not performed, the location of stained cells at crypt base was similar (Fig. 5) in accordance with previous study. ${ }^{17}$ This suggests that CD44 and LGR5-positive cells may be involved in early gastric carcinogenesis. We did not observe any correlation between LGR5 IHC and expression of mRNA in the present study. The transcriptional result may not discriminate the spatial difference and could include all parts of expression. Interestingly, immunoreactivity of LGR5, known for the stem cell marker of antrum, was detected in gastric body with IM. Although we did not demonstrate that IM arises from CSCs, our data can be considered as preliminary evidence that provides further insights into the possibility that the pathogenesis of IM is associated with LGR5 or CSCs.

CD44, most studied, is a cell surface transmembrane glycoprotein encoded by the CD44 gene, a 20-exon DNA segment, ${ }^{18}$ of which exons 1-5 and 16-20 are spliced together and translated into CD44s, the standard isoform. The variant exons 6-15 can be alternatively spliced and assembled in different combinations from the standard exons to generate other variant protein isoforms (CD44v). Although CD44 has been recognized as a CSC marker in several types of cancer, ${ }^{19}$ the ubiquitous expression of CD44 in many cell type, ${ }^{20}$ reduce the usefulness of CD44s as a CSC marker. Moreover, the existence of alternatively spliced variants may be the reason for the conflicting result of CD44, which was implicated in both tumor suppression and progression. ${ }^{21,22}$ In a genetic mice model for gastric carcinogenesis, the mouse homolog of human CD44v8-10 is expressed in precancerous regions of the stomach, from which tumor-initiating cells are thought to arise, suggesting that CD44v8-10 may be a marker for gastric tumor-initiating cells in the mouse. ${ }^{23}$

We showed a step-wise increase in CD44 immunostaining in the normal, dysplasia, and cancer sequence $(P$ trend $<0.001)$ indicating $H$. pylori infection may promote initiation of tumorigenesis via gastric CSCs. However, CD44 mRNA did not show any correlation with IHC analysis of CD44. Although CD44 mRNA expressed strongly in control subjects compared to cancer patients, expression of CD44v8-10 mRNA in cancer patients was higher than control subjects, suggesting that CD44v8-10 was a specific marker for gastric CSCs, but not CD44. ${ }^{6,7}$ In this concept,
CD44v8-10 mRNA seems more appropriate for the gastric CSC marker. Nonetheless, since there is still no significant correlation between CD44 IHC and any of CD44 or variant form of CD44 gene expression in the present study, CD44-stained cells in tissue could include normal stem cells. More study still needs to validate the use of CD44v8-10 mRNA in unpurified cells or tissue level.

The present work has several limitations. Since we did not perform functional study for confirming stem cell properties by cell culture or animal models, it is not clear whether the elevated number of CD44 or LGR5-positive epithelial cells reflects true gastric CSCs. Although the downregulation of LGR5 and CD44 IHC was observed after $H$. pylori eradication, the expression of CD44 v8-10mRNA was not. Moreover, since mucosal resection was performed in the dysplasia and cancer groups, the reduction of LGR5 IHC in mucosal base at follow-up cannot be solely attributable to $H$. pylori eradication.

Nonetheless, this is one of the few studies that evaluated the clinical meaning of LGR5 and CD44 expression in tissue as a potential gastric CSC marker by compare its expression in normal, dysplasia, and cancer groups and before and after $H$. pylori eradication. Moreover, we measured and compared the expression of mRNA of both CD44v8-10 and CD44, providing result that the possibility of CD44v8-10 as a more useful gastric CSC marker than CD44.

In conclusion, LGR5 and CD44 IHC results could be used as probable gastric CSC markers to evaluate the development of gastric cancer as probable gastric CSCs in tissue. Regarding transcriptional level, CD44v8-10 may be more suitable for CD44 or LGR5 in gastric CSC researches. More studies are needed to identify the association between CD44 IHC and CD44v8-10 mRNA expression.

\section{ACKNOWLEDGMENTS}

This work was supported by a National Research Foundation (NRF) of Korea grant for the Global Core Research Center (GCRC) funded by the Korea government (MSIP) (No. 2011-0030001).

\section{CONFLICTS OF INTEREST}

No potential conflicts of interest were disclosed.

\section{REFERENCES}

1. Jemal A, Siegel R, Ward E, Hao Y, Xu J, Murray T, et al. Cancer statistics, 2008. CA Cancer J Clin 2008;58:71-96. 
2. Takaishi S, Okumura T, Wang TC. Gastric cancer stem cells. J Clin Oncol 2008:26:2876-82.

3. Choi YJ, Kim N, Chang H, Lee HS, Park SM, Park JH, et al. Helicobacter pylori-induced epithelial-mesenchymal transition, a potential role of gastric cancer initiation and an emergence of stem cells. Carcinogenesis 2015:36:553-63.

4. Mani SA, Guo W, Liao MJ, Eaton EN, Ayyanan A, Zhou AY, et al. The epithelial-mesenchymal transition generates cells with properties of stem cells. Cell 2008;133:704-15.

5. Levi E, Sochacki P, Khoury N, Patel BB, Majumdar AP. Cancer stem cells in Helicobacter pylori infection and aging: Implications for gastric carcinogenesis. World J Gastrointest Pathophysiol 2014; 5:366-72.

6. Schmittgen TD, Livak KJ. Analyzing real-time PCR data by the comparative C(T) method. Nat Protoc 2008;3:1101-8.

7. Lau WM, Teng E, Chong HS, Lopez KA, Tay AY, Salto-Tellez M, et al. CD44v8-10 is a cancer-specific marker for gastric cancer stem cells. Cancer Res 2014;74:2630-41.

8. Barker N, van Es JH, Kuipers J, Kujala P, van den Born M, Cozijnsen $\mathrm{M}$, et al. Identification of stem cells in small intestine and colon by marker gene Lgr5. Nature 2007;449:1003-7.

9. Sigal M, Rothenberg ME, Logan CY, Lee JY, Honaker RW, Cooper RL, et al. Helicobacter pylori activates and expands Lgr5(+) stem cells through direct colonization of the gastric glands. Gastroenterology 2015:148:1392-404.e21.

10. Kim N, Park YS, Cho SI, Lee HS, Choe G, Kim IW, et al. Prevalence and risk factors of atrophic gastritis and intestinal metaplasia in a Korean population without significant gastroduodenal disease. Helicobacter 2008;13:245-55.

11. Dixon MF, Genta RM, Yardley JH, Correa P. Classification and grading of gastritis. The updated Sydney System. International Workshop on the Histopathology of Gastritis, Houston 1994. Am J Surg Pathol 1996;20:1161-81.

12. Ryu HS, Park DJ, Kim HH, Kim WH, Lee HS. Combination of epithelial-mesenchymal transition and cancer stem cell-like phenotypes has independent prognostic value in gastric cancer. Hum Pathol 2012;43:520-8.

13. van Diest PJ, van Dam P, Henzen-Logmans SC, Berns E, van der
Burg ME, Green J, et al. A scoring system for immunohistochemical staining: consensus report of the task force for basic research of the EORTC-GCCG. European Organization for Research and Treatment of Cancer-Gynaecological Cancer Cooperative Group. J Clin Pathol 1997:50:801-4.

14. Clarke MF, Dick JE, Dirks PB, Eaves CJ, Jamieson CH, Jones DL, et al. Cancer stem cells: perspectives on current status and future directions: AACR Workshop on cancer stem cells. Cancer Res 2006:66:9339-44.

15. Wu C, Xie Y, Gao F, Wang Y, Guo Y, Tian H, et al. Lgr5 expression as stem cell marker in human gastric gland and its relatedness with other putative cancer stem cell markers. Gene 2013;525:18-25.

16. Jang BG, Lee BL, Kim WH. Distribution of LGR5+ cells and associated implications during the early stage of gastric tumorigenesis. PLoS One 2013;8:e82390.

17. Simon E, Petke D, Böger C, Behrens HM, Warneke V, Ebert M, et al. The spatial distribution of LGR5 + cells correlates with gastric cancer progression. PLoS One 2012;7:e35486.

18. Screaton GR, Bell MV, Jackson DG, Cornelis FB, Gerth U, Bell JI. Genomic structure of DNA encoding the lymphocyte homing receptor CD44 reveals at least 12 alternatively spliced exons. Proc Natl Acad Sci U S A 1992;89:12160-4.

19. Zöller M. CD44: can a cancer-initiating cell profit from an abundantly expressed molecule? Nat Rev Cancer 2011;11:254-67.

20. Collado A, de Andres A, Cañadas E, Ruiz-Cabello F, Gomez O, Pedrinaci S, et al. Characterization of CD44 antigen during lymphoid ontogeny. Immunobiology 1991;183:1-11.

21. Sheridan C, Kishimoto H, Fuchs RK, Mehrotra S, Bhat-Nakshatri P, Turner $\mathrm{CH}$, et al. CD44+/CD24- breast cancer cells exhibit enhanced invasive properties: an early step necessary for metastasis. Breast Cancer Res 2006;8:R59.

22. Lopez JI, Camenisch TD, Stevens MV, Sands BJ, McDonald J, Schroeder JA. CD44 attenuates metastatic invasion during breast cancer progression. Cancer Res 2005;65:6755-63.

23. Ishimoto T, Oshima H, Oshima M, Kai K, Torii R, Masuko T, et al. CD44+ slow-cycling tumor cell expansion is triggered by cooperative actions of Wnt and prostaglandin E2 in gastric tumorigenesis. Cancer Sci 2010;101:673-8. 